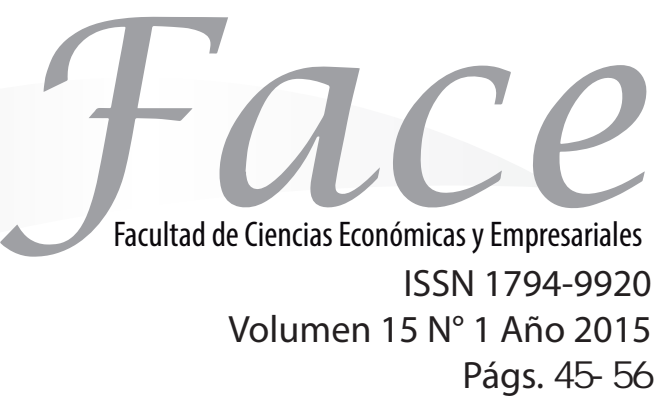

\title{
ESTRATEGIAS FINANCIERAS PARA EL MANEJO DE LOS PASIVOS CIRCULANTES EN LAS EMPRESAS PRIVADAS DEL SECTOR COMERCIAL DEL MUNICIPIO MIRANDA
}

\author{
Milangela del Carmen, Romero Velásquez* \\ Luis Emiro, Belloso** \\ Derber José, Soto Rodríguez $z^{* * *}$ \\ Ángel Alberto, Nava Chirinos***** \\ Misleida Coromoto, Nava Chirinos***** \\ Roland Antonio, Nava Chirinos $* * * * * *$ \\ María Francisca, Nava de Guere $* * * * * * * *$ \\ Albis Gabriela, Oldenburg Paz Pa****** $^{* * *}$
}

Fecha de Recepción: 12 de Febrero 2015

Fecha de Aprobación: 29 de Mayo 2015

\section{Resumen:}

El propósito del estudio fue determinar las estrategias financieras para el manejo de pasivos circulantes en las empresas del sector comercial del municipio Miranda. Las teorías consultadas fueron Block y Hirt (2005), Gitman (2007), Moyer (2005), Ortiz (2005), Van (2007). La metodología es de tipo descriptivo no experimental - transeccional con una población de (46) Gerentes, utilizándose un cuestionario de 30 ítems escala tipo Lickert, obteniendo una confiabilidad de 0.91. Los resultados determinaron la necesidad de fijar políticas a través de un plan de contabilidad que garantice el uso racional de los recursos bajo el resguardo del marco legal que así lo establece.

PALABRAS CLAVE: Estrategias Financieras, Pasivos Circulantes, Empresas Comerciales 


\title{
FINANCIAL STRATEGIES FOR THE MANAGEMENT OF THE COMMERCIAL SECTOR COMPANY'S CURRENT LIABILITIES IN THE MUNICIPALITY OF MIRANDA
}

\begin{abstract}
:
The purpose of the research was to determine the financial strategies for the management of the commercial sector company's current liabilities in the municipality of Miranda. The study was based on the theoretical principles of Block and Hirt (2005), Gitman (2007), Moyer (2005), Ortiz (2005) and Van (2007). A descriptive non experimental cross-sectional methodology was used for a population of 46 managers, and a 30 item scale Likert questionnaire with a reliability of 0.91 was administered. The results showed the need to identify an accounting plan that guarantees a rational use of resources within a legal framework.
\end{abstract}

Keywords: FinancialStrategies, Current Liabilities, Commercial Business

\section{ESTRATÉGIAS FINANCEIRAS PARA O GERENCIAMENTO DO PASIVO CIRCULANTE NAS EMPRESAS DO SETOR COMERCIAL DO MUNICÍPIO MIRANDA.}

\begin{abstract}
Resumo:
O objetivo doestudo foi determinarasestratégias financeiraspara o gerenciamento dopasivo circulantenas empresasdo setor comercialdo municípioMiranda.O trabalho basou-se nas teorias de Block e Hirt(2005),Gitman(2007), Moyer(2005), Ortiz(2005), e Van(2007). Utilizou-se uma metodologia descritivanãoexperimental-transeccionalcom uma população de(46)gerentes, e se aplicou um questionário com 30 itens a escala tipo Linker com uma confiabiliade de 0.91.Os resultados indicaram a necessidade deestabelecer políticasatravés de um planode contabilidade quegaranta o usoracional dos recursossob um resguardo juridico.
\end{abstract}

Palavras- Chave: Estratégias Financeiras, Passivo Circulante, Empresas Comerciais.

\footnotetext{
* Lcda. en Administración / MSc. Gerencia Financiera. U.N.E. Rafael María Baralt UNERMB / Universidad Dr. Rafael Belloso Ch Contacto: milangelaromerov@gmail.com

** Licdo. Administración de Empresas / MSc. Gerencia Financiera. U.N.E. Rafael María Baralt UNERMB / Universidad Dr. Rafael Belloso Ch Contacto: luis_belloso@hotmail.com.

**** Contador Público / Maestrante en Gerencia Financiera. Universidad de la Guajira UNIGUAJIRA / Universidad de Pamplona Contacto: delbersoto@hotmail.com

**** Licdo. Administración / MSc. Gerencia RRHH/ Dr. Ciencias Gerenciales. U.N.E. Rafael María Baralt UNERMB / Universidad Dr. Rafael Belloso Ch. Contacto: angelnavach66@hotmail.com

***** Licda. Administración / MSc. Gerencia RRHH/ Dra. Ciencias Gerenciales. U.N.E. Rafael María Baralt UNERMB

Contacto: misleida@hotmail.com

******Roland Antonio, Nava Chirinos. Licdo. en Administración. U.N.E. Rafael María Baralt UNERMB. Contacto: rolandnava63@hotmail.co

******* Licda. En Educación / Especialista en Ciencias Sociales. Contacto: marucha2458@hotmail.com

******** Lcda. en Administración / MSc. Gerencia Financiera

U.N.E. Rafael María Baralt UNERMB. Contacto: albisoldenburg@yahoo.es
} 
Milangela del Carmen, Romero Velásquez - Luis Emiro, Belloso - Derber José, Soto Rodríguez - Ángel Alberto, Nava Chirinos - Misleida Coromoto, Nava Chirinos - Roland Antonio, Nava Chirinos - María Francisca, Nava de Guere - Albis Gabriela - Oldenburg Paz

\section{Introducción:}

El dinamismo experimentado en el orden económico mundial ante el proceso de globalización, ha propiciado cambios en las organizaciones que tienen por finalidad el uso de sus recursos y factor humano, para lo cual han realizado grandes esfuerzos orientados a evaluar la calidad, productividad y rentabilidad de sus negocios, para alcanzar niveles óptimos en sus procesos productivos y lograr el liderazgo en el mercado creciente.

Al respecto, en las últimas décadas se ha observado el rápido crecimiento de las empresas desafiando la ingenuidad de los administradores financieros para proporcionar un financiamiento adecuado. Incluso, las ventas que se expanden rápidamente pueden ocasionar una presión intensa en las acumulaciones de inventarios y de las cuentas por cobrar, con lo que se agotan los recursos de la empresa. De igual manera, puede existir el exceso de flujo de efectivo, sin que haya una óptima utilización del mismo.

Esta situación obliga a los gerentes a buscar soluciones y decisiones financieras para mejorar su procesos, donde los sistemas de costos son útiles, pues, reflejan los datos relacionados con la producción; adicionalmente, también involucran la preparación de estimados de costos y elaboración de presupuestos, como herramienta fundamental para el control y para una mejor planificación de los fondos en el presupuesto de inversión de dichas compañías.

En tal sentido, Block y Hirt (2005) plantean, la administración de capital de trabajo implica el financiamiento y la administración de los activos circulantes de la empresa. Así mismo, es probable que el gerente financiero dedique más tiempo a la administración del capital de trabajo que a cualquier otra actividad. Por otra parte, Ortiz (2005) señala que al administrador el capital de trabajo debe primar el objetivo de evitar los extremos relacionados con la saturación o insuficiencia de fondos por concepto de caja y bancos, cartera e inventario dadas sus implicaciones sobre la carga de costos. En consecuencia, conviene garantizar una posición de fuerte liquidez para asegurar la operación, pero, es pertinente considerar que una excesiva liquidez ejerce efectos negativos sobre la movilización de los recursos invertidos y sobre la rentabilidad.
Es importante señalar que a los gerentes debe preocupar sustancialmente, el manejo de los recursos de factible inversión o comprometidos en activos fijos, no por ello puede desconocerse que en algunas empresas se asignan pequeños fondos a la inversión que conforman el capital de trabajo bruto correspondiente a cuentas por cobrar, inventario, inversiones temporales, caja y bancos.

En este sentido Perdomo (2000), destaca que al gerencial el capital de trabajo intervienen cursos de acción que definen los pasos a implementar para lograr los objetivos establecidos para el activo circulante y el pasivo a corto plazo, es decir, se definen acciones para tomar decisiones, denominadas estrategias financieras explicadas de manera detallada en las bases teóricas de este proyecto.

En este orden de ideas, las organizaciones Privadas del Sector Comercial en Venezuela presentan una escasa utilización de las fuentes de financiamiento a causa de pensamientos errados y además cultura organizacional equivocada por parte del personal directivo, todo esto trae como consecuencia, una mala utilización de los recursos disponibles. En este sentido las empresas estudiadas presentan un excesivo flujo de efectivo en banco, debido a la desconfianza y a la desmotivación con respecto a factores económicos, políticos y sociales del país, generando como consecuencias desventajas a favor de la competencia, además de una administración inadecuada.

\section{Marco teórico:}

\subsection{Estrategias financieras}

Según Francés (2005), "son las que emergen, cuando surgen las acciones emprendidas, bien formuladas que permiten canalizar los esfuerzos y asignar los recursos de una organización, y se adopta una posición singular y viable, basada en las capacidades internas (fortalezas, debilidades) anticipando los cambios en el entorno y posibles movimientos del mercado y de sus competidores (oportunidades y amenazas)".

En este sentido Gitman (2007) señala, las estrategias financieras son las metas, patrones 0 alternativas trazadas en áreas de perfeccionar la gestión financiera de una empresa con un fin predeterminado, el de mejorar los resultados existentes para alcanzar o acercarse a los óptimos, 
mediante la generación de valor para la organización. Es decir, las estrategias financieras ayudan a desarrollar un proceso para asegurar la sostenibilidad financiera de la organización.

Al respecto Van (2007), la estrategia financiera comprende tres elementos fundamentales que las organizaciones deben considerar al momento de tomar alguna decisión; indicadores a corto plazo, el proceso de inversión y los tipos de estrategias financieras; cada una de éstas debe relacionarse con los objetivos de la organización; la combinación óptima de las tres maximizará el valor de la organización para los accionistas. Aunado a esto, agrega que el crecimiento de las empresas contemporáneas, las mismas se convierte en un vasto agregado de tareas que desempeñan un cuerpo de empleados especializados, generalmente de alta preparación.

En relación a la definición antes señalada, las Estrategias Financieras es el conjunto de procedimientos, técnicas, otros que emergen en la medida en que las acciones que se quieren emprender y las cuales deben estar planeadas para poder canalizar todos los recursos disponibles que posee la empresa internamente son las fortalezas que constituyen ventajas competitivas y las debilidades que son deficiencias en el desempeño o cumplimiento de sus objetivos, es decir, son obstáculos o limitaciones que mediante las estrategias aplicadas se puede mejorar la posición competitiva.

Por otra parte, las Estrategias Financieras, permiten anticiparse a los cambios suscitados en el entorno en cuanto a oportunidades, los cuales serán los factores que representan una posibilidad de mejorar la organización para aprovecharlas al máximo y las amenazas, las cuales son elementos perturbadores que pudieran perjudicar o limitar el desempeño de la empresa en lo que al mercado y competidores se refiere, caso del presente estudio las pequeñas y medianas empresas existentes en el Municipio Miranda, del Estado Zulia.

Para finalizar las estrategias financieras empresariales deben estar en correspondencia con la estrategia maestra que se haya decidido a partir del proceso de planeación estratégica de la organización. Consecuentemente, cada estrategia deberá llevar el sello distintivo que le permita apoyar el cumplimiento de la estrategia general y con ello la misión y los objetivos estratégicos.

\subsection{Pasivos Circulantes}

El pasivo circulante de una empresa está formado por sus deudas a corto plazo, que deben ser pagadas en un plazo inferior a los doce meses. Se trata, por lo tanto, del pasivo exigible a corto plazo que es circulante ya que no existe intención de que permanezca en la empresa durante mucho tiempo y está en constante rotación o movimiento.

Según Villegas (2008,) el pasivo se clasifica de acuerdo con la fecha de vencimiento o liquidación en pasivo a corto plazo (menos de un año). Todos los pasivos representan un compromiso que la empresa tiene y que deberá cubrir en el período en curso o en períodos futuros.

Igualmente, el pasivo a corto plazo o circulante representa todas las obligaciones, que para ser cubiertas requieren, dentro del período en curso, el uso de algún activo o la creación de otro pasivo. Normalmente, está relacionado con la operación normal de la empresa y se encuentra en constante movimiento o rotación.

Básicamente está constituido a criterio del autor antes citado por las siguientes partidas:

- Cuentas por Pagar a Proveedores: Esta cuenta se forma por el financiamiento que los proveedores otorgan en forma de mercancías. Así mismo constituye un pasivo fundamental para la existencia de cualquier empresa, ya que a través de éste la empresa obtiene mercancía que transforma y vende.

- Préstamos Bancarios: Esta cuenta está formada por préstamos que hacen los bancos para el pago de inventarios o para financiar cuentas por cobrar (clientes que le deben a la empresa)

- Impuestos por Pagar: Por lo general, las empresas quedan debiendo los impuestos que devengan; este adeudo es lo que constituye el saldo de esta cuenta. Los impuestos pueden ser: impuesto sobre la renta, impuesto al valor agregado, impuestos municipales, retención de impuesto sobre la renta, impuestos aduaneros, entre otros.

En este mismo orden de ideas, Van (2007) plantea que las empresas deben establecer políticas de pagos por compras, mano de obra, impuestos y otros gastos. Como resultado, las cuentas por pagar $y$ las acumulaciones representan variables de decisiones pasivas; las decisiones varían de acuerdo 
Milangela del Carmen, Romero Velásquez - Luis Emiro, Belloso - Derber José, Soto Rodríguez - Ángel Alberto, Nava Chirinos - Misleida Coromoto, Nava Chirinos - Roland Antonio, Nava Chirinos - María Francisca, Nava de Guere - Albis Gabriela - Oldenburg Paz

con los cambios en el nivel de la producción o servicios ofrecidos $y$, en el caso de los impuestos por pagar, con un cambio en los ingresos antes de impuestos. En cierto sentido, este componente de los pasivos circulantes representa un financiamiento incorporado, tiende a elevarse con las ampliaciones de la empresa y a bajar en las contracciones.

Cabe señalar que, si los mercados financieros fueran imperfectos o incompletos, los accionistas se beneficiarían por el hecho de que las empresas empacaran sus instrumentos de deuda en tal forma que aprovecharan estas circunstancias. Las imperfecciones que más afectan el financiamiento por deuda son los costos de flotación, los de quiebra, los de información y las restricciones sobre los acreedores.

Entre los pasivos más conocidos según Van (2007) se encuentran:

- Cuentas por Pagar: Es una forma de financiamiento de corto plazo común a casi todos los negocios; es la mayor fuente de fondos de manera colectiva. En una economía avanzada no se exige que la mayoría de los compradores paguen por sus bienes a la entrega, sino que se permite un corto período de espera antes de vencerse el pago. Durante ese período el vendedor de los bienes extiende crédito al comprador, puesto que los proveedores generalmente son más liberales en la extensión de crédito que las instituciones financieras: las pequeñas empresas en especial dependen de las cuentas por pagar o crédito comercial.

- Gastos y Cargos Acumulados por Pagar: Representan una fuente espontánea de financiamiento. Las cuentas acumuladas más comunes son los salarios e impuestos; para ambas se incurre en o se acumula en el gasto, pero no se paga. Generalmente se especifica una fecha que indica cuando se debe pagar la acumulación. Al igual que las cuentas por pagar las acumulaciones tienden a ampliarse con el alcance de la operación; a medida que aumentan las ventas los costos de mano de obra suelen aumentar y con ellos, los salarios; al incrementarse las utilidades aumentan los impuestos acumulados.

En tal sentido las acumulaciones representan financiamiento sin costo y sin intereses, ya que no se paga hasta el final o después del final del período de pago, la compañía establece el lapso, de igual manera sucede con los impuestos, no se pagan hasta su fecha de vencimiento. No obstante, una empresa debe tener cuidado extremo en la postergación de los salarios, debe informar plenamente a los empleados y fijar una fecha firme para su pago.

- Préstamos Bancarios: Es un préstamo comercial con un vencimiento original o final de más de un año que se paga de acuerdo con un programa específico; en la mayoría de los casos estos préstamos se cubren en abonos periódicos (trimestrales, semestrales o anuales). Este programa de pago suele estar relacionado con la capacidad del flujo de efectivo del prestatario para dar servicio a la deuda; este programa demanda abonos iguales periódicos, pero puede especificar cantidades irregulares o su pago total a su vencimiento.

- Papeles Comerciales: El mercado del papel comercial está compuesto de dos partes: el mercado del corredor y el mercado de colocación directa. Las empresas industriales, las de servicios públicos y empresas financieras de tamaño mediano venden papeles comerciales por medio de corredores. La organización de los corredores está compuesta de media docena de corredores principales que compran papel comercial al emisor y a su vez lo venden a los inversionistas.

Cabe destacar que cierto número de empresas financieras de ventas pasan por alto la organización de los corredores con el fin de vender sus documentos directamente a los inversionistas.

- Préstamos Internacionales: Si una empresa está expuesta en la moneda de un país determinado, puede pedir prestado en ese país para compensar la exposición. Tanto los activos monetarios como los pasivos monetarios tienen un coeficiente de exposición de 1.0, de manera que sirven como equilibrio. Existen una gran variedad de fuentes de financiamiento externo para la filial extranjera, éstas van desde préstamos de bancos comerciales dentro del país anfitrión hasta agencias de préstamos internacionales. 


\subsection{Principales Fuentes De Financiamiento}

\section{Externo}

Préstamos en bancos comerciales y facturas comerciales: Son una de las principales fuentes $y$ desempeñan esencialmente la misma función que los bancos locales, a diferencia de que las prácticas bancarias en Europa permiten préstamos a plazos más largos que los que se pueden obtener en Estados Unidos, inclusive, tienden a ser sobre una base de sobregiro.

Financiamiento mediante eurodólares: Se define como eurodólar al depósito en dólares de un banco fuera de Estados Unidos; se ha desarrollado un mercado activo para estos depósitos desde fines de los cincuenta. Los bancos extranjeros y las sucursales extranjeras de bancos estadounidenses en especial en Europa negocian activamente depósitos en eurodólares pagando tasas de interés que fluctúan de acuerdo con la oferta y la demanda.

Financiamiento mediante bonos: El mercado de eurodivisas debe distinguirse del mercado de eurobonos, el segundo es más tradicional y se caracteriza porque los suscriptores colocan valores, no obstante la emisión de un bono puede estar nominada en una sola divisa.

Bonos de opción de divisas y de divisas múltiples: Algunos bonos otorgan a su tenedor el derecho de escoger la moneda en que se recibe el pago, usualmente antes de cada pago de cupón o del principal. Esta opción suele estar restringida a dos monedas, aunque pueden ser más.

Finalmente, Moyer y otros (2005) definen los pasivos circulantes de la siguiente manera:

- Crédito Comercial: Normalmente una empresa recibe este tipo de pasivo cuando el proveedor le entrega la mercancía que solicitó y le permite un lapso específico antes de pagarla; es la fuente de financiamiento a corto plazo más importante para las empresas mercantiles. Es utilizada como fuente para financiar operaciones en las pequeñas empresas, ya que a menudo no están en condiciones de obtener fondos de bancos u otras fuentes de financiamiento en los mercados financieros.

- Gastos Devengados e Ingreso Diferido: Son otras fuentes espontáneas de crédito a corto plazo sin garantía, como ejemplos de estos están los salarios, impuestos e intereses, aplazamiento del pago de comisiones de ventas y bonificaciones; del mismo modo, los intereses devengados por una emisión de bonos puede servir como fuente de financiamiento por períodos de hasta seis meses, en consecuencia representan una fuente de financiamiento libre de intereses.

- Crédito Bancario: Inicialmente, los bancos comerciales proporcionan otra fuente importante de crédito a corto plazo con y sin garantía lo que permite satisfacer las necesidades estacionales de fondos de las compañías, como financiar la acumulación de inventarios y cuentas por cobrar. No obstante, cuando una compañía obtiene un crédito, por lo común firma un pagaré en donde se especifica el monto del préstamo, la tasa de interés que se cobrará y la fecha de vencimiento.

- Papeles Comerciales: Consiste en pagarés a corto plazo sin garantía emitidos por grandes compañías; solo las empresas con altas calificaciones de crédito pueden obtener fondos en préstamo mediante la venta de papeles comerciales. Así mismo, estos papeles son adquiridos en la mayoría de los casos por empresas con fondos excedentes, como bancos, compañías de seguros, fondos de pensiones, fondos de inversión de mercado de dinero y otras instituciones financieras.

Por otra parte Gitman (2007), hace referencia a los préstamos internacionales y señala que la obtención de esta fuente de financiamiento a corto plazo no es diferente del financiamiento de las operaciones puramente nacionales, ya que en ambos casos se deben financiar la fabricación y el almacenamiento de productos para la venta y continuar después con el financiamiento de las cuentas por cobrar antes de recibir cualquier pago en efectivo de las ventas.

No obstante, esta modalidad es muy distinta del comercio nacional; principalmente, los pagos se realizan o reciben en moneda extranjera. Otra característica es que es mayor el tamaño y la fecha de vencimiento, por tanto, las empresas que realizan 
Milangela del Carmen, Romero Velásquez - Luis Emiro, Belloso - Derber José, Soto Rodríguez - Ángel Alberto, Nava Chirinos - Misleida Coromoto, Nava Chirinos - Roland Antonio, Nava Chirinos - María Francisca, Nava de Guere - Albis Gabriela - Oldenburg Paz

este tipo de operación tienen que financiar mayores cantidades de dólares durante períodos de tiempo más largos que las empresas que operan a nivel nacional.

\section{Metodología:}

La investigación es de tipo descriptiva, en tal sentido, Hernández y Otros (2003) señalan que los estudios descriptivos miden, evalúan o recolectan datos sobre diversos aspectos, dimensiones 0 componentes. Asimismo, opinan que las investigaciones transaccionales descriptivas tienen como objetivo indagar la incidencia y los valores con que se manifiestan una $O$ más variables. El procedimiento consiste en medir un grupo de persona u objetos y proporcionar sus descripciones comparativas entre grupos o subgrupos de personas, objeto o indicadores.La investigación se enmarco bajo el perfil de un diseño de campo - no experimental -transeccional que según Hernández y otros (2003) considera no experimental de campo, ya que las variables de estudio no fueron manipuladas presentándose tal y como se muestran en el contexto de análisis, siendo así un estudio de campo caracterizándose por que la información o data es recolectada de la realidad misma donde se suceden los hechos.

En este sentido, Sierra (2004) señala que los estudios no experimentales no comprenden diversidad de observaciones, ni de grupos, ni tampoco variables experimentales, quedan limitados a una sola observación, de un solo grupo en un único momento de tiempo, sin intervenir en ellos, ni manipularlo, son diseños simples, las más frecuentes indagaciones.

La poblacion del presente estudio está constituida por todas las empresas privadas del sector comercial dedicadas a la venta de artículos para el hogar y uso personal, en el municipio Miranda. No obstante, Parra (2004) hace referencia a que la poblaciones el conjunto conformado por todos los elementos, seres u objetos que contienen la característica y menciones u observaciones que se requieren en una investigación dada.

Cabe señalar que Parra (2004), describe la población como el conjunto integrado por todas las mediciones $\mathrm{u}$ observaciones de interés en el universo. Por lo tanto pueden definirse varias poblaciones en un solo universo, tantas como

característica a medir. En el caso de la presente investigación, la población quedó conformada por los Gerentes/ Administradores de cuarenta y seis (46) empresas privadas del sector comercial del municipio Miranda.De allí, la población quedó constituida por los gerentes de finanzas $\mathrm{y} / \mathrm{o}$ administradores, de las 46 empresas comerciales que se encuentran registradas, según puede observarse en el siguiente cuadro. Ahora bien, Por lo pequeño y accesible de la población no se recurrió a establecimiento de una muestra, sino que se procedió a trabajar con toda la población (es decir se recurrió a la técnica del censo poblacional).

Tabla $N^{\circ} 1$.

Cuadro de distribución de la Población

Universo:

Empresas

3

4

6

$$
7
$$

$$
8
$$

8

$$
10
$$

10 Bain Huerta Murray

\&Asociados,C.A. (B.H.M.

11 Constructora JesusDubc, C.A. (Jeduca).

12 Constructora Leon $\mathrm{Y}$ Polanco,C.A. (Colpoco).

13 Constructora Tropicana,C.A.

14 Barrio Romero Servicios $E$ Inverciones, C.A. (Barsinca)

15 Barrios Hidalgo

Construcciones,S.A. (Bahiconsa).

16 C \& R Consultores Gerenciales S.C.

17 C.A. De Servicios Multiples

18 C.A TecnoeletricasCabimas (Cateca)

Unidades
Informantes:
Cargo de los
Informantes
Gerente
Administrador
Gerente
encargado
Administrador
Gerente
Administrador
Gerente
Administrador
Administrador
Administrador
Gerente
encargado
Administrador
Admistrador
Administrador
Gerente
Administrador
Admistrador




\begin{tabular}{|c|c|c|}
\hline 19 & C.A. Vencemos. & $\begin{array}{l}\text { Gerente } \\
\text { Administrador }\end{array}$ \\
\hline 20 & C.A. Vigilantes Del Zulia & Administrador \\
\hline 21 & $\begin{array}{l}\text { Fabricaciones Aislantes } \\
\text { Venezolana,C.A. (Faiveca). }\end{array}$ & $\begin{array}{l}\text { Gerente } \\
\text { Administrador }\end{array}$ \\
\hline 22 & $\begin{array}{l}\text { Consorcio } \\
\text { Socoven\&SocovenIngeneria. }\end{array}$ & $\begin{array}{l}\text { Gerente } \\
\text { Administrador }\end{array}$ \\
\hline 23 & $\begin{array}{l}\text { Consorcio Socoven, C. A.P.C } \\
\text { I. }\end{array}$ & Administrador \\
\hline 24 & Consorcio Textuline, C.E. C.A. & Gerente \\
\hline 25 & ConstratistaCoquivacoa, C.A. & Administrador \\
\hline 26 & $\begin{array}{l}\text { Construcciones Altagracia,C.A. } \\
\text { (Conalca) }\end{array}$ & Gerente \\
\hline 27 & $\begin{array}{l}\text { Construcciones Civiles } \\
\text { Electricas Y Mecanica,S.A. } \\
\text { (Cocemsa) }\end{array}$ & $\begin{array}{l}\text { Gerente } \\
\text { Administrador }\end{array}$ \\
\hline 28 & $\begin{array}{l}\text { Construcciones } \\
\text { Dalmonte,C.A.(Condalca) }\end{array}$ & Administrador \\
\hline 29 & $\begin{array}{l}\text { Construcciones E } \\
\text { Intalaciones,C.A .(Coninsca) }\end{array}$ & $\begin{array}{l}\text { Gerente } \\
\text { Administrador }\end{array}$ \\
\hline 30 & $\begin{array}{l}\text { Construcciones E } \\
\text { InvercionesHym. }\end{array}$ & Gerente \\
\hline 31 & $\begin{array}{l}\text { Construcciones E Inversiones } \\
\text { Altagracia. }\end{array}$ & Administrador \\
\hline 32 & $\begin{array}{l}\text { Construcciones Intalaciones Y } \\
\text { Servicios,C.A. (Cisca) }\end{array}$ & $\begin{array}{l}\text { Gerente } \\
\text { Administrador }\end{array}$ \\
\hline 33 & $\begin{array}{l}\text { Fabricas De Ventanas De } \\
\text { Aluminio,C.A. (Favenalum). }\end{array}$ & $\begin{array}{l}\text { Gerente } \\
\text { Administrador }\end{array}$ \\
\hline 34 & $\begin{array}{l}\text { Construcciones Lavera, } \\
\text { C.A.(Conlaviera). }\end{array}$ & Administrador \\
\hline 35 & $\begin{array}{l}\text { Construcciones } \\
\text { Mantenimiento Servicios } \\
\text { Electricos,C.A.(Comaselca) }\end{array}$ & Gerente \\
\hline 36 & $\begin{array}{l}\text { Construcciones } \\
\text { MejiaGelvez,C.A. }\end{array}$ & Administrador \\
\hline 37 & Construcciones Monserca,S.A. & $\begin{array}{l}\text { Gerente } \\
\text { Administrador }\end{array}$ \\
\hline 38 & $\begin{array}{l}\text { Construcciones Nava \& Araujo, } \\
\text { C.A. (Conarauca). }\end{array}$ & Gerente \\
\hline 39 & $\begin{array}{l}\text { Construcciones } Y \\
\text { Mantenimiento Buena } \\
\text { Vista,C.A. }\end{array}$ & Administrador \\
\hline 40 & $\begin{array}{l}\text { Construcciones } Y \\
\text { Mantenimientos A \& S, C. } \\
\text { A.(Comanasca) }\end{array}$ & $\begin{array}{l}\text { Gerente } \\
\text { Administrador }\end{array}$ \\
\hline 41 & $\begin{array}{l}\text { Construcciones Y } \\
\text { Mantenimientos A \& S, C. } \\
\text { A.(Comanasca) }\end{array}$ & $\begin{array}{l}\text { Gerente } \\
\text { Administrador }\end{array}$ \\
\hline 42 & $\begin{array}{l}\text { Construcciones Y Servicios J.E. } \\
\text { C.A. }\end{array}$ & Administrador \\
\hline 43 & $\begin{array}{l}\text { Construcciones Y Servicios } \\
\text { Miranda,C.A. (Consermica) }\end{array}$ & $\begin{array}{l}\text { Gerente } \\
\text { Administrador }\end{array}$ \\
\hline 44 & $\begin{array}{l}\text { Construcciones Y Servicios } \\
\text { San Antonio. (Consersanca). }\end{array}$ & Gerente \\
\hline 45 & $\begin{array}{l}\text { Construcciones } \\
\text { Zuliana,C.A.(Const-Zul) }\end{array}$ & Administrador \\
\hline 46 & Constructora Cordova, C.A & Gerente \\
\hline & $\begin{array}{l}\text { Fuente: Alcaldía del Municipio. } \\
\text { Miranda (2008). }\end{array}$ & \\
\hline
\end{tabular}

Cabe señalar que el instrumento diseñado es el cuestionario (sin intervención del encuestador) debido a las características de la población en estudio, coincidiendo con lo señalado por Méndez (2007) quien plantea, el cuestionario supone su aplicación a una población bastante homogénea con variables similares y problemática semejante, para ser respondido o llenado por los administradores, encargados o directores del área de las empresas.

No obstante, para Hernández y otros (2003) consiste en una serie de preguntas respecto a una o más variables a medir. Para la elaboración de un cuestionario se cuenta con las diferentes modalidades de preguntas, abiertas y cerradas, las primeras no delimitan las alternativas de respuestas y las posibilidades de respuestas son infinitas, mientras que las segundas son fáciles de codificar y preparar, para su análisis.

Una vez elaborado el instrumento, el mismo, se sometió a un estudio técnico para la identificación de su validez de contenido. Según Hernández y otros (2003) la confiabilidad de un instrumento de medición se refiere al grado en que su aplicación repetida al mismo sujeto u objeto produce resultados iguales. En este sentido los autores definen validez como el grado en que un instrumento realmente mide la variable que pretende medir.

Dentro de este marco, Méndez (2007) refiere que para que un cuestionario sea confiable debe medir el rasgo o rasgos que intenta estimar. Construir las preguntas adecuadas es fundamental para la calidad de la información que el investigador requiere. Así mismo el citado autor nos dice que este aspecto de la validez es importante y deseable en un trabajo de investigación, y garantiza al investigador que la información obtenida podrá ayudar en su propósito.

De allí pues, que para el caso especifico de esta investigación, la validez del cuestionario, se obtuvo mediante su evaluación por parte de cinco (5) expertos en el área, quienes luego de ser diseñado, revisaron la validez de los ítems con respecto a la variable, dimensiones e indicadores establecidos y emitieron juicios respecto a la redacción y contenido verificando si los mismos median las variables y sus respectivos indicadores, 
Milangela del Carmen, Romero Velásquez - Luis Emiro, Belloso - Derber José, Soto Rodríguez - Ángel Alberto, Nava Chirinos - Misleida Coromoto, Nava Chirinos - Roland Antonio, Nava Chirinos - María Francisca, Nava de Guere - Albis Gabriela - Oldenburg Paz

así como los objetivos de la investigación, emitiendo comentarios en un instrumento de pertinencia.

Señalan Hernández y otros (2003), que existen diversos procedimientos para calcular la confiabilidad de un instrumento de medición. Todos utilizan fórmulas que producen coeficientes de nivel de confianza que oscilan entre 0 y 1 , donde un coeficiente de 0 significa nula confiabilidad y 1 representa un máximo de confiabilidad. Es decir que en cuanto el coeficiente se acerque más a 0 mayor error habrá y todo lo contrario cuando el coeficiente se acerque a 1.

Según Parra (2004) el nivel de confianza (confiabilidad) es la probabilidad de que la estimación efectuada se ajuste a la realidad. Cualquier información que queremos recoger esta distribuidita según la ley de probabilidad. Así llamamos nivel de confianza a la probabilidad de que el intervalo construido en torno a un estadístico capte el verdadero valor del parámetro.

En otro orden de ideas, según Méndez (2007) una vez que se construye el cuestionario, se debe validar la confiabilidad dentro de lo que se llama prueba piloto o pretest, el cual se aplicara en personas que posean las mismas características de las personas de la población o muestra identificada. Por lo cual, se determinará el análisis y discriminación de ítems aplicando el pretest a una muestra de 10 sujetos de similares características a las de la población objeto de estudio, con el fin de buscar la factibilidad de uso del instrumento.

Por otra parte, se procedió a realizar el cálculo de la confiabilidad del instrumento a través de la aplicación del cálculo de coeficiente de confiabilidad Alfa-Cronbach calculado a través del programa Excel y la siguiente fórmula:

$$
r=\frac{K}{K-1}\left[1-\frac{\sum S_{i}^{2}}{S_{t}^{2}}\right]
$$

Donde:

$$
r=\text { coeficiente alfa Cronbach }
$$

$\mathrm{K}=$ número de ítemes del instrumento

$$
S_{i}^{2}=\text { sumatoria de las varianzas de los }
$$

puntajes de cada ítems

$S_{t}^{2}=$ varianza de los puntajes totales

Despejando la formula se obtiene, para el instrumento COM-AR-2009:

$$
\begin{gathered}
r=\frac{30}{30-1}\left[1-\frac{23.650}{214.092}\right] \\
\left.=\frac{30}{29}[1-0.1105]=1.01790 .8895\right] \\
r=0.91
\end{gathered}
$$

Cabe destacar que la información procesada dependió de cada una de las respuestas de las interrogantes planteadas, es por ello, que se utilizó el análisis cuantitativo que a juicio de Hernández y Otros (2003) comprende la observación de los datos numéricos derivados de la exploración para determinar las conclusiones necesarias, todo esto incluye tabulación, organización y tratamiento de la información.

El cálculo estadístico idóneo para este estudio, de acuerdo al tipo de diseño será el de estadística descriptiva definida por Hernández y Otros (2003) como la técnica poco compleja, empleada en estudios descriptivos cuya finalidad depende de la profundidad establecida por el investigador, considerando el nivel de medición y la utilización de distribuciones de frecuencia absoluta. 
Tabla 2.

\section{Dimensión Manejo de los Pasivos Circulantes}

\begin{tabular}{|c|c|c|c|c|c|c|c|c|c|c|}
\hline $\begin{array}{l}\text { Categoría de } \\
\text { Respuestas }\end{array}$ & & & & 气ू & & $\frac{\text { के }}{\frac{2}{2}}$ & & & \multirow{3}{*}{ 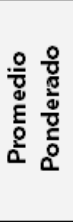 } & \multirow[t]{3}{*}{ 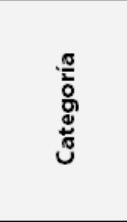 } \\
\hline \multirow{2}{*}{ Indicadores } & \multicolumn{2}{|c|}{1} & \multicolumn{2}{|c|}{2} & \multicolumn{2}{|c|}{3} & \multicolumn{2}{|c|}{4} & & \\
\hline & Fa. & $\%$ & Fa. & $\%$ & Fa. & $\%$ & Fa. & $\%$ & & \\
\hline $\begin{array}{l}\text { Cuentas y efectos por } \\
\text { pagar }\end{array}$ & 1 & 2,17 & 23 & 50,00 & 22 & 47,83 & 0 & 0,00 & 2,46 & $\begin{array}{c}\text { Mod. } \\
\text { Deficiente }\end{array}$ \\
\hline $\begin{array}{l}\text { Gastosy cargos } \\
\text { acumulados por pagar }\end{array}$ & 0 & 0,00 & 22 & 47,83 & 23 & 50,00 & 1 & 2,17 & 2,54 & $\begin{array}{c}\text { Mod. } \\
\text { Deficiente }\end{array}$ \\
\hline Préstamos Bancarios & 1 & 2,17 & 22 & 47,83 & 23 & 50,00 & 1 & 2,17 & 2,54 & $\begin{array}{c}\text { Mod. } \\
\text { deficiente }\end{array}$ \\
\hline Papeles comerciales & 0 & 0,00 & 24 & 52,17 & 22 & 47,83 & 0 & 0,00 & 2,48 & $\begin{array}{c}\text { Mod. } \\
\text { Deficiente }\end{array}$ \\
\hline $\begin{array}{l}\text { Préstamos } \\
\text { internacionales }\end{array}$ & 0 & 0.00 & 0 & 0.00 & 0 & 0.00 & 0 & 0.00 & 0.00 & $\begin{array}{l}\text { Sin } \\
\text { opinión }\end{array}$ \\
\hline TOTAL & 1 & 0,72 & 69 & 50,00 & 67 & 48,55 & 1 & 0,72 & 2,49 & $\begin{array}{c}\text { Mod. } \\
\text { Deficiente }\end{array}$ \\
\hline
\end{tabular}

\section{Resultados y discusión:}

Se presentan los resultados obtenidos a través de la aplicación del instrumento diseñado para conocer el comportamiento de la variable por medio de su dimensión, manejo de los pasivos circulantes, el cual resulto Moderadamente Deficiente, atendiendo lo reflejado por la puntuación alcanzada por el Promedio Ponderado $(X=2,49)$, evidenciándose en los valores de los indicadores: cuentas y efectos por pagar $(X=2.46)$ con una categoría moderadamente deficiente el cual refleja las limitaciones que presentan las empresas objeto de estudio al hacer uso de sus cuentas por pagar al momento de tomar decisiones; gastos $y$ cargos acumulados por pagar $(X=2,54)$ obteniendo una categoría moderadamente deficiente el cual permite inferir que las empresas estudiadas no manejan este tipo de indicador vislumbrándose el desconocimiento por parte de los gerentes y administradores de estas empresas sobre las consecuencias que esta mala praxis puede ocasionar, préstamos bancarios $(\mathrm{X}=2.54)$ arrojando una categoría moderadamente deficiente lo que indica que los gerentes de las empresas estudiadas no aplican este tipo de indicador, ya que consideran que existen muchos trámites administrativos para la solicitud de estos préstamos bancarios; papeles comerciales $(X=2,48)$ con una categoría de moderadamente deficiente lo cual refleja el desconocimiento por parte de los gerentes o administradores para utilizar este indicador en la toma de decisiones; y los préstamos internacionales $(X=00.00)$ con una categoría sin opinión lo que indica que estas empresas no manejan y no ponen en práctica este tipo de estrategias en sus operaciones.

En consecuencia Villegas (2008), señala que el manejo del los pasivos circulantes se vinculan con el corto plazo, porque representa todas las obligaciones que una empresa u organización posee, y que para ser cubiertas requieren, dentro del período en curso, el uso de algún activo o la creación de otro pasivo. Habitualmente, está relacionado con la operación normal de la empresa y se encuentra en constante movimiento o rotación. 
Milangela del Carmen, Romero Velásquez - Luis Emiro, Belloso - Derber José, Soto Rodríguez - Ángel Alberto, Nava Chirinos - Misleida Coromoto, Nava Chirinos - Roland Antonio, Nava Chirinos - María Francisca, Nava de Guere - Albis Gabriela - Oldenburg Paz

Se pudo observar que las cuentas por pagar que manejan las empresas estudiadas no han sido lo suficientemente eficaces para las mismas, en este sentido se encontraron debilidades y limitaciones que obstaculizan el buen manejo de las actividades.

Las empresas estudiadas deben vigilar que no se vayan arrastrando faltantes en provisiones mensuales registradas, ajustándose su monto a la fecha en que se realiza su pago respectivo, produciéndose diferencias resultantes entre la estimación efectuada y el pago realizado. Estas diferencias deben ajustarse en el momento que se conocen para que la administración tenga una información actualizada. Uno de los indicadores más comunes en los gastos y cargos acumulados son las nóminas que implican pagos significativos, las cuales influyen en forma importante en el capital de trabajo. Su control se inicia en la autorización para contratar empleados y obreros hasta su pago en los periodos correspondientes.

El grupo de empresas privadas comerciales evitan hacer uso de los créditos bancarios, lo que no les permite avanzar en el crecimiento de las mismas. Estas evidencian el descontento por parte de los gerentes 0 administradores para diligenciar los trámites administrativos para la solicitud de este tipo de estrategias.

Las empresas estudiadas consideran poco importante el uso de papeles comerciales para obtener fondos que permitan la consecución de las actividades en desarrollo que emprenden las mismas. En consecuencia estas empresas no manejan las letra de cambio y los pagaré para tomar decisiones en un momento determinado, considerándose estos documentos de gran importancia, ya que los mismos conforman una garantía de recuperación del valor de la prestación de un servicio o de la venta de algún bien, ya sea mueble o inmueble; puesto que mediante su emisión, el tomador puede recurrir a fuentes legales establecidas para hacer efectivo su pago.

Para finalizar las estrategias en el manejo de pasivos circulantes de las empresas privadas del sector comercial del municipio Miranda, son utilizadas de manera inadecuada de manera que se hace necesario fijar políticas a través de un plan de contabilidad que garantice el uso racional y eficiente de los recursos bajo el resguardo del marco legal que así lo establece. Tal determinación obedece a las limitaciones encontradas en la gestión que desempeñan las empresas en materia de las cuentas y efectos por pagar, gastos y cargos administrativos por pagar, préstamos bancarios, papeles comerciales $y$ en cuanto a los préstamos internacionales estos no son utilizados dada las condiciones legales que la regulan.

Aunado a estas conclusiones, se agrega que tales beneficios no se producen por la falta de procesos de trabajo documentados y el desinterés de los trabajadores por realizar el trabajo en función de los lineamientos de los accionistas.

\section{Referencias:}

Block, S y Hirt, G (2005). Administración Financiera. México: Editorial Mc Graw-Hill.

Francés, A (2005). Estrategias para la empresa en América Latina. Caracas - Venezuela. Editorial ISEA.

Gitman, L (2007). Principios de Administración Financiera. México: Editorial Addison Wesly.

Hernández, R.; Fernández, H.; Baptista, J. (2003). Metodología de la Investigación. Caracas: Editorial Mc Graw - Hill.

Méndez, C. (2007) Metodología de la investigación, Editorial Biblioteca Central Universidad Central de Venezuela, Caracas, Venezuela.

Moyer, Ch (2005). Administración Financiera Contemporánea. México: Editorial Thompson.

Ortiz, A. (2005). Gerencia Financiera y Diagnostico Estratégico. McGraw Hill. Colombia.

Parra, J. (2004) Guía de muestreo. Colección XLV Aniversario FACES. $2^{\text {a }}$ edición. Editorial de La Universidad del Zulia. 
Perdomo, A (2000). Análisis e Interpretación de Estados Financieros. México D.F. Editorial Thonsom

Sierra, R (2004) Técnicas de Investigación Social. España: Editorial Paraninfo.

Van, J y Wachowicz, J. (2007). Administración Financiera. Prentice Hall. México.

Villegas, R (2008) La Capitalización Empresarial. Editorial CENDES. Caracas, Venezuela. 